\title{
Theoretical Approach in Vp/Vs Prediction from Rock Conductivity in Gas Saturating Shaly Sand
}

\author{
Denny MF. Mendrofa ${ }^{1}$ \\ ${ }^{1}$ Doctoral Student of Petroleum Engineering, Institut Teknologi Bandung Jl. Ganesa No.10, Bandung, West Java, \\ Indonesia \\ Correspondence: Denny MF. Mendrofa, Petroleum Engineering, Institut Teknologi Bandung Jl. Ganesa No.10, \\ Bandung, West Java, Indonesia. Email: dmendrofa@yahoo.com
}

Received: Sep. 17, 2018

doi:10.5539/mas.v13n1p45

\author{
Accepted: Sep. 25, 2018 \\ Online Published: December 10, 2018 \\ URL: https://doi.org/10.5539/mas.v13n1p45
}

\begin{abstract}
Most of study in Oil and Gas Industry are studying Vp/Vs behaviour against hydrocarbon presence in a porous rock. Vp/Vs number is commonly used to model Amplitude Variation against Offset response of a gas sand which allow us be able to discriminate it from the water sand. The model is built in term to match the synthesized Vp/Vs against the observed $\mathrm{Vp} / \mathrm{Vs}$ which actually correspond to elastic property of porous and fluid saturating rocks. This study is aimed to find correlation between elastic property and conductivity of saturated rocks, especially reservoir in this study is found as a shaly sand unit, a turbidite sand deposit in Kutai Basin, East Kalimantan. The correlation between elastic property and conductivity properties are rarely discussed in many studies, however this study gives a new insight and evidence of how elastic and the inverse of conductivity (resistivity) properties are correlating both ways formulized theoretically with a support from Gassmann and Archie equations. In this study, more realistic condition is accomplished by taking clay mineral into account in sand unit and hence impact to $\mathrm{Vp} / \mathrm{Vs}$ derivation from resistivity. Furthermore, sand-shale texture is considered important when this study giving a significant discrepancy of how clay mineral is distributed in sand unit and impacted to $\mathrm{Vp} / \mathrm{Vs}$ and resistivity values. Thomas-Stieber diagram is useful when defining a disperse and/or laminate distribution of shale in the observed porous sand.
\end{abstract}

Keywords: rock physics, Gassmann's equation and assumption, sand-shale texture, Thomas-Stieber diagram, effective medium model

\section{Introduction}

\subsection{Background}

The behaviour of seismic wavespropagation in porous rocks can reflect the properties of the rocks based on the elastic properties of porous rocks which are commonly saturated by fluid. The nature of this elasticity of rocks isstudied by Gassmann (1951) who observed the ability of saturated rocks to restore its initial shape due to mechanical disturbances in the form of seismic waves. In the Gassman's formulate the amount of fluid content and the pores of rocks affect the rapid wave propagation through acoustics media. The shale distribution structure in the formation also contributes to the speed of the compressed wave (P-wave).

In rock physics study, predicting sand shale texture from seismic is quite rare reviewed. Homogenity, single mineralogy assumption and isotropic rock have been bearing in concept when attempting to do forward modeling properly in practice (Avseth, Mukerji \& Mavko, 2005), but unfortunately the importance of rock texture is slightly ignored and can bring error to predict the elastic modulus sand hence yielded propagating error in predicting acoustic velocity.

Rock physics is an important link between seismic data with the presence of in-situ hydrocarbons and reservoir characteristics. Rock Physics Template presents the relationship among seismic attribute, petrophysical parameter, and geological model in one curve. Based on the concept presented, the RPT shows that Vp/Vs correlates to AI (Acoustic Impedance) which is influenced by large porosity and saturation of rock fluid. A research on analytical relationship between AI (Acoustic Impedance) and resistivity is completed by Mendrofa (2006) and gave a deeper insight of how resistivity is correlabe to acoustic impedance. The fluid saturation inside the rock pores is a form of resistivity rock function (Archie, 1941). In clean sand rocks, pure resistivity reflects the fluid response, thus the 
response of resistivity will have a similar trend to the $\mathrm{Vp} / \mathrm{Vs}$ trend.

Gassmann's formulation is developed from Hooke's law and used to study the effect of stiffness of porous medium which allows compressional and shear waves propagating along medium within following assumption and limitation:

- Low seismic frequencies are assumed, so that pore pressures are equilibrated throughout the pore space. Insitu seismic conditions should generally be acceptable. Ultrasonic laboratory conditions will generally not be well-described with Gassmann's equation. Sonic-logging frequencies may or may not be within the range of validity, depending on the rock type and the fluid viscosity;

- The rock is homogeneous and isotropic;

- All minerals making up the rock have the same bulk and shear modulus;

- Fluid-bearing rock is completely saturated.

Hence, the objectivess of this research are to establish a modification on Gassmann's equation for shaly sand through analytical approach which can model $\mathrm{Vp} / \mathrm{Vs}$ against resistivity log response, analyze sensitivity factor of the new analytical equation, and observe mineral complexity by defining shale sand texture against acoustic wave.

\section{Method}

The used method outline in this study is arranged in Fig.1.

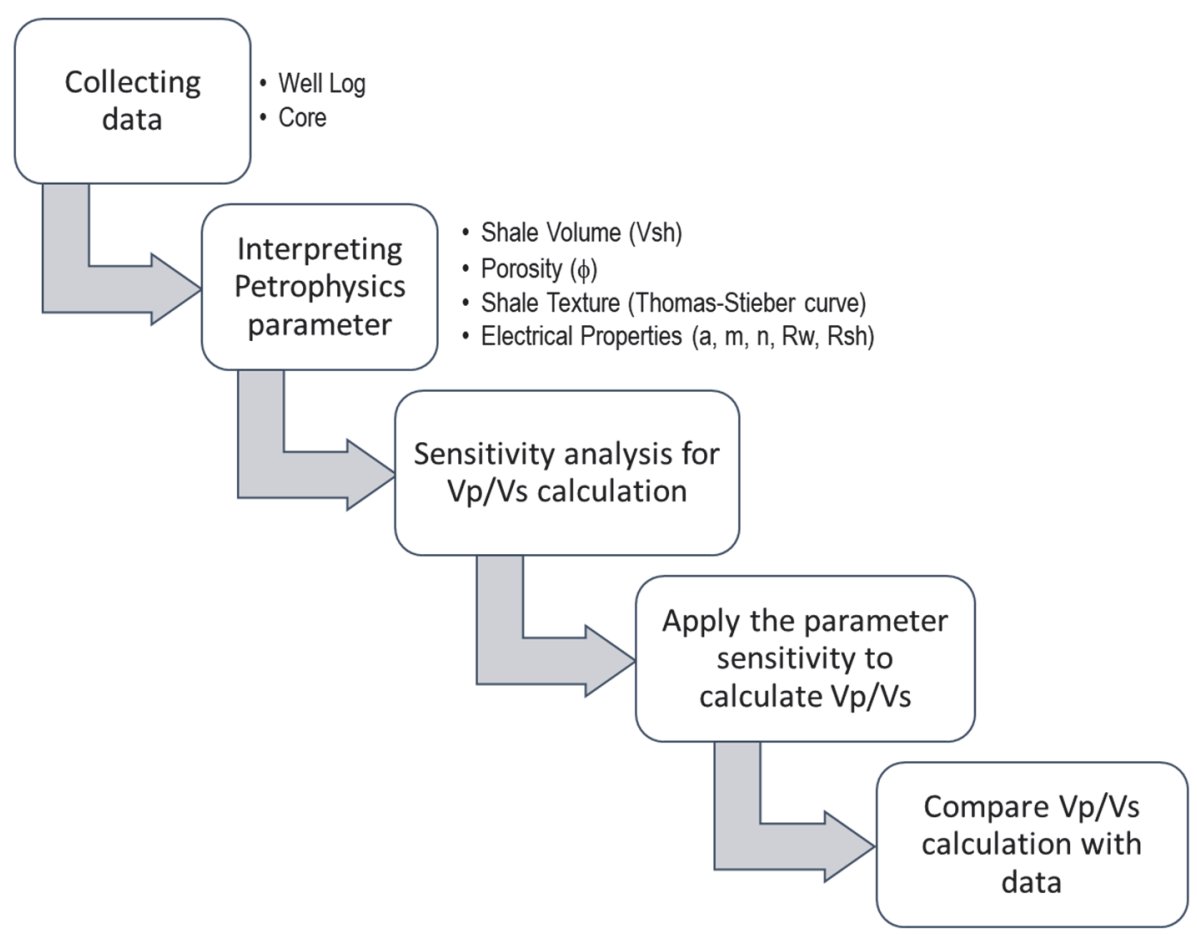

Figure 1. Research Methodology

In this research, core data are used to validate the petrophysical analysis especially in sand shale texture analysis. $\mathrm{P}$-wave and S-wave velocity ratios are calculated using analytical equation which can be an alternative method to calculate $\mathrm{Vp} / \mathrm{Vs}$ when only well $\log$ data is available. Then, the result of calculation is compared with P-wave and S-wave velocity ratios from sonic log to prove the analytical approach.

In order to get the best assumption elastic modulus parameters, this research builds an analyzer tool to do the sensitivity easily. The analyzer sensitivity tool is shown in the following figure. 


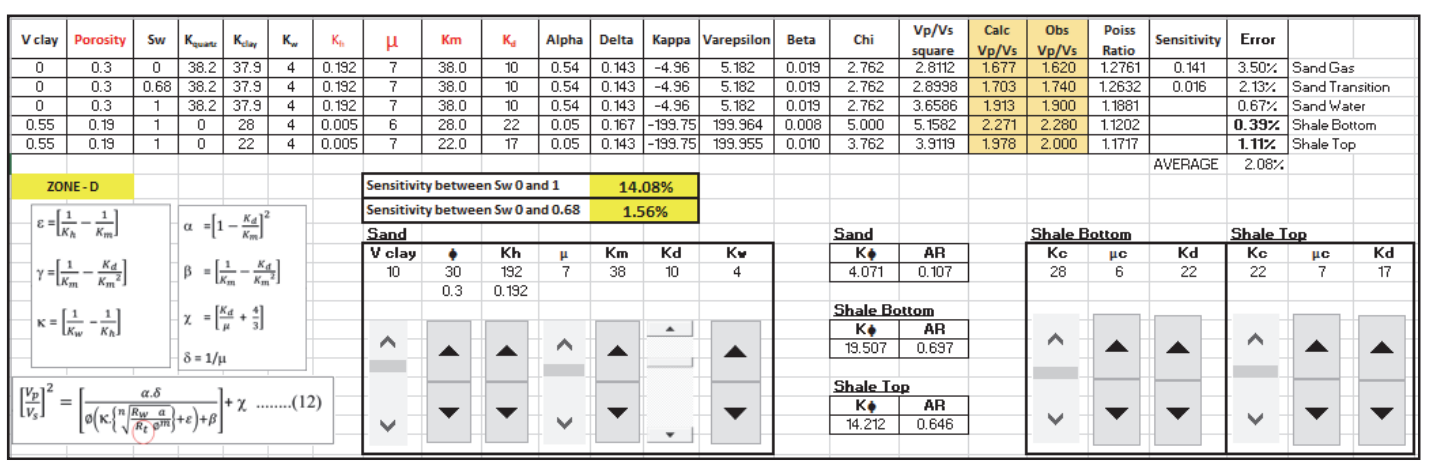

Figure 2. Analyzer Tool for Scanning Reservoir Parameter

\subsection{A New Analytical Approach}

An analytical approach is derived from Gassmann's equation so far asthe most widely used method to predict seismic velocity in various fluid saturation in reservoir as seismic data are commonly used to explore reservoir. Gassmann's formulation is developed from Hooke's law and used to study the effect of stiffness of porous medium that allows compressional and shear waves propagating along medium. The Gassmann's equation is:

$$
\begin{gathered}
V_{p}^{2}=\frac{K_{\text {sat }}+4 / 3 \mu}{\rho} \\
V_{p}^{2} \rho=K_{\text {sat }}+4 / 3 \mu \\
=\left(K_{d}+\Delta K_{d}\right)+4 / 3 \mu \\
=\left[K_{d}+K_{f} \frac{\left(1-K_{d} / K_{m}\right)^{2}}{\left(1-K_{f} / K_{m}\right) \emptyset+\left(K_{m}-K_{d}\right)^{K} / K_{m}{ }^{2}}\right]+\frac{4}{3} \mu
\end{gathered}
$$

Where,

$$
K_{f}^{-1}=S_{w}\left(K_{w}\right)^{-1}+S_{h}\left(K_{h}\right)^{-1}
$$

While,

$$
V_{s}^{2} \rho=\mu
$$

For any density, Vp/Vs can be written as (Mendrofa, 2006):

$$
\begin{aligned}
& {\left[\frac{V_{p}}{V_{s}}\right]^{2}=\frac{\left(\left[K_{d}+K_{f} \frac{\left(1-{ }^{K_{d} / K_{m}}\right)^{2}}{\left(1-{ }^{K} / K_{m}\right) \varnothing+\left(K_{m}-K_{d}\right)^{K_{f}} / K_{m}{ }^{2}}\right]+\frac{4}{3} \mu\right)}{\mu}} \\
& {\left[\frac{V_{p}}{V_{s}}\right]^{2}=\frac{\left[K_{f} \frac{\left(1-{ }^{K} \frac{K_{d} / K_{m}}{}\right)^{2}}{\left(1-{ }^{K} / K_{m}\right) \varnothing+\left(\frac{1}{K_{m}}-\frac{K_{d}}{K_{m}}\right)^{2} K_{f}}\right]}{\mu}+\frac{K_{d}}{\mu}+\frac{4}{3}}
\end{aligned}
$$

Substitute Eq. (4) to Eq. (6), then Eq. (6) becomes:

$$
\left(\frac{V_{p}}{V_{s}}\right)^{2}=\frac{\left(1-\frac{K_{d}}{K_{m}}\right)^{2} \frac{1}{\mu}}{\left[S_{w}\left(\frac{1}{K_{w}}-\frac{1}{K_{h}}\right)+\frac{1}{K_{h}}-\frac{1}{K_{m}}\right] \emptyset+\left(\frac{1}{K_{m}}-\frac{K_{d}}{K_{m}^{2}}\right)}+\left(\frac{K_{d}}{\mu}+\frac{4}{3}\right)
$$

Some variables in Eq. (7) can be separated and grouped into following formula: 


$$
\left(\frac{V_{p}}{V_{s}}\right)^{2}=\frac{\alpha \cdot \delta}{\left[\left(\kappa \cdot S_{w}\right)+\varepsilon\right] \phi+\beta}+\chi
$$

Where,

$$
\begin{gathered}
\alpha=\left(1-\frac{K_{d}}{K_{m}}\right)^{2} \\
\delta=\frac{1}{\mu} \\
\kappa=\frac{1}{K_{w}}-\frac{1}{K_{h}} \\
\varepsilon=\frac{1}{K_{h}}-\frac{1}{K_{m}} \\
\beta=\frac{1}{K_{m}}-\frac{K_{d}}{K_{m}{ }^{2}} \\
\chi=\frac{K_{d}}{\mu}+\frac{4}{3}
\end{gathered}
$$

Hence, Eq. (8) becomes an obvious formula whenVp/Vs square is the inverse ofSw and this reality can emphasize the previous hypothesis of RPT crossplot due to Vp/Vs relationship between Acoustic Impedance and gas saturation $\left(1-\mathrm{S}_{\mathrm{w}}\right)$ accordingly.

Moreover, another follow up work is required when Sw must be calculated from rock resistivity. From this point, another hypothesis arise when rock resistivity has an an indirect relationship with $\mathrm{Vp} / \mathrm{Vs}$.

Archie (1941) definess water saturation from Formation Resistivity Factor (FF) and Resistivity Index (RI),

$$
F F=\frac{R_{o}}{R_{w}}=\frac{a}{\emptyset^{m}}
$$

Any bulk resistivity is is normalized to rockssaturatedby water(Resistivity Index),

$$
R I=\frac{R_{t}}{R_{o}}=\frac{1}{S_{w}{ }^{n}}
$$

Then, water saturation is written in an Archie's equation for clean sand,

$$
S_{w}=\sqrt[n]{\frac{a \cdot R_{w}}{\emptyset^{m} \cdot R_{t}}}
$$

A modified Archie's equation is introduced whenamount of shale volume are embedded in pore fill which gives,

$$
\begin{gathered}
S_{w_{-} \text {disp }}=\sqrt[n]{\left(\frac{1}{R_{t}}-\frac{V_{s h}}{R_{s h}}\right) \cdot \frac{a \cdot R_{W}}{\emptyset^{m}\left(1-V_{s h}\right)}} \\
S_{w_{-} \text {lam }}=\sqrt[n]{\left(\frac{1}{R_{t}}-\frac{V_{s h}{ }^{2}}{R_{s h}}\right) \cdot \frac{a \cdot R_{w}}{\emptyset^{m}}}
\end{gathered}
$$

Combining these two equations (Gassmann and Archie) to water saturation as a hub for both approach that can give a theoretical prediction in deriving acoustic and shear velocity directly from conductivity property of saturated porous medium. From Eq.(8), Eq.(11)\& Eq.(12), Vp/Vs relationship with Rock Resistivity can be formulized 
straightly in:

$$
\left(\frac{V_{p}}{V_{s}}\right)^{2}=\frac{\alpha \cdot \delta}{\left[\left(\kappa \cdot S_{w}\right)+\varepsilon\right] \phi+\beta}+\chi
$$

We can obtain porosity, constant a (tortuosity factor), $\mathrm{m}$ (cementation factor), $\mathrm{n}$ (saturation exponent), resistivity of water, and porosity from petrophysical analysis. However, further validation will be taken care to prove the relation by analyzing carefully on determination of petrophysical parameter $\left(K_{d}, K_{f}, K_{m}, K_{g}, K_{w}\right.$, a-m-n, $R_{s h}, V_{s h}$, and $\mathrm{R}_{\mathrm{w}}$ ) and with variables of $\phi$ and $\mathrm{R}_{\mathrm{t}}$ in simplified rock complexity, saturated with gas \& water.

\subsection{Mixing Law}

Single mineral element is rarely found in real data which can meet Gassmann's requirement of homogenous rock, hence complex mineralogy requiress an average (single value) of elastic modulus to be determined by observing carefully:

- Fraction of individual mineral

- Modulus (inverse of compressibility) of each individual mineral

- Pore shape (geometry)

The best method for the average property is mixing law. For that reason, a flow diagram below is defined to calculate average modulus of homogenized mineral.

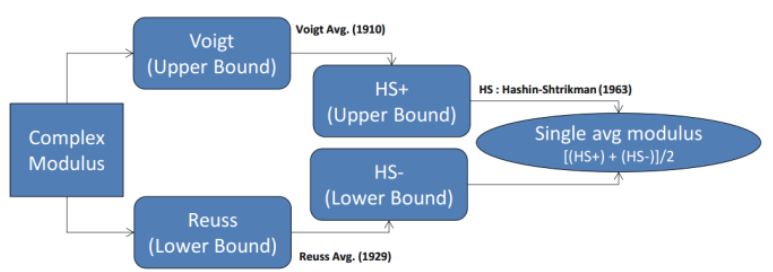

Figure 3. Flow diagram - homogenizing a complex medium

The simplest bounds conceptis introduced by Voigt (1910) and Reuss (1929). The Voigt upper bound on the effective elastic modulus, $M_{v}$, of a mixture of $N$ minerals in the medium is,

$$
M_{v}=\sum_{i=1}^{N} f_{i} M_{i}
$$

The Reuss lower bound of the effective elastic modulus, $M_{\mathrm{R}}$, is

$$
\frac{1}{M_{R}}=\sum_{i=1}^{N} \frac{f_{i}}{M_{i}}
$$

The elastic modulus of rock is the average of Voight and Reuss:

$$
K_{m}=\frac{M_{v}+M_{R}}{2}
$$

where $f_{i}$ is the volume fraction of the $\mathrm{i}^{\text {th }}$ element, and $M_{i}$ is the elastic modulus of the $\mathrm{i}^{\text {th }}$ element. The Voigt bound is sometimes called the isostrain average as it provides the ratio of average stress to average strain when all constituents are assumed to have the same strain. While the Reuss bound is sometimes called the isostress average as it gives the ratio of average stress to average strain when all constituents are assumed to have the same stress (Avseth, Mukerji, \& Mavko, 2005).

The Reuss average illustrates exactly the effective modulus of grain solid suspension in a fluid. In addition, Reuss average is also the basic for describing certain types of elastic sediments and for illustrating the modulus of "shattered" materials in which solid fragments are completely surrounded by pore fluid. The presence of a gas or liquid with a zero shear modulus on the medium, Reuss average will provide an effective modulus of the mixture 
(Mavko, Mukerji, \& Dvorkin, 2009).

\subsection{Thomas-Stieber Method}

Porosity, volume of shale and water saturation, is the main needed parameter for resource estimation of sand layers. Those are interdependent on each other. However, the problem is that all three parameters are affected by the shale distribution in the pores. There are three types of shale distributions in sand:

1. Laminated-layer of shale within the sand.

2. Dispersed shale on sand grains or pore filling.

3. Shale particles sized structural sandsized in load-bearing position within the rock.

The shale distribution and porosity can be computed form Thomas-Stieber cross-plot, in which volume of shale is plotted on X-axis and total porosity on Y-axis. Tyagi et. al. (2009) makes a formula to create Thomas-Stieber plot.

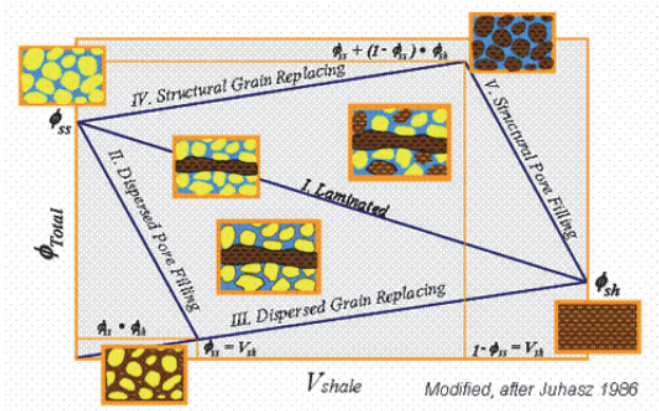

Figure 4. Thomas-Stiebershale distribution model(Tyagi, Guha, Voleti, \& Saxena, 2009)

From Figure 4, the laminated bound is created from sand porosity with zero clay volume to shale porosity with $100 \%$ percent of clay volume. While the dispersed bound is drawn from sand porosity with zero clay volume to sand porosity in clay volume scale (x-axis) to the point which is equal to sand porosity multiplied by shale porosity in $y$-axis. Then, the actual data is plotted to the Thomas-Stieber bound to analyze the type of shale distribution in sand layer.

\subsection{Petrophysic and Core Data}

The used data for validation is extracted from petrophysical analysis. There are four wells data which are employed in this case, $\mathrm{X}-21, \mathrm{X}-7, \mathrm{X}-1$, and $\mathrm{X}-8$. A preliminary analysis indicates the selected real data is taken from deepwater turbidite sedimentary rock which dominantly consists of sandstone with quartz \& clay as major components. A shaly sand reservoir in a normal pressure \& temperature gradient against depth is filled with gas water system and with presence of fluid contact available at some places. There were 20 cores which are drawn in X-8 well.

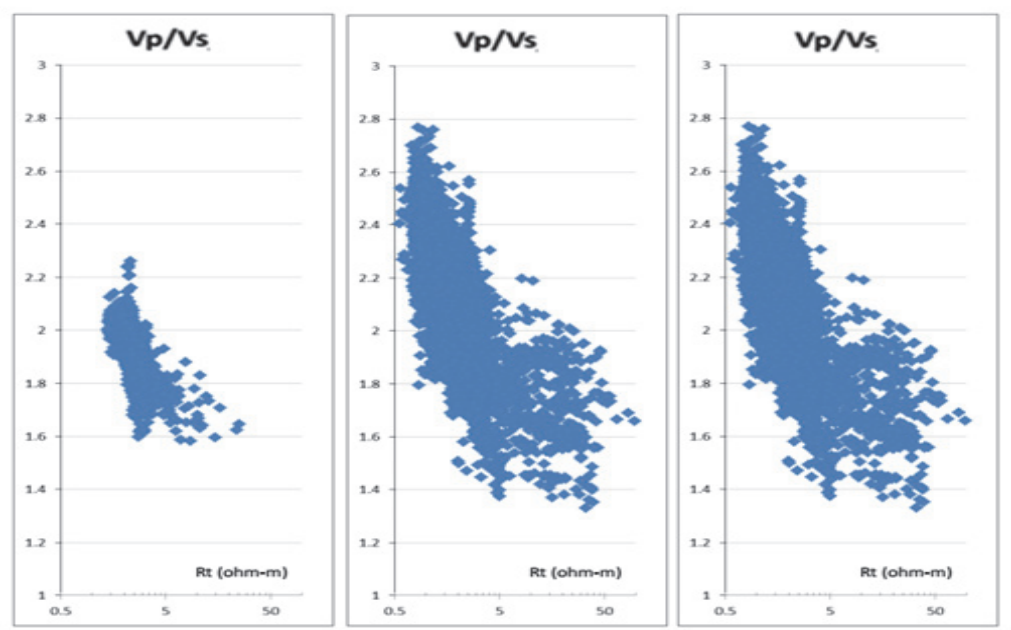

Figure 5. Crossplot between P-wave and S-wave velocity ratios and rock resistivity from several zones 


\section{Results}

Based on Eq. (10), it shows that seismic velocity can be linked up with petrophysical parameters such as: porosity, resistivity, tortuosity factor, cementation exponent, and saturation exponent. However, fraction and elastic modulus of each constituent are the most considered parameter rather than pore geometry when it is required to simplify the mathematical establishment. However, all elastic properties whether it is mineral modulus or fluid modulus are influenced by petrophysical parameters.

Fig.5 shows that the plot between $\mathrm{Vp} / \mathrm{Vs}$ and resistivity has a unique trend. It proves that the analytical equation which is provided in this study is sound. So, we can calculate P-wave and S-wave velocity ratios by employing well log data.

Gassmann's equation mostly consistss of elastic modulus:

- $\mathrm{K}_{\mathrm{d}}$ is calculated when fluid is extracted out from the pore space.

- $\mathrm{K}_{\mathrm{f}}$ consistss of gas and water in dual phase system and its presence tend to soften the stiffness.

- $\mathrm{K}_{\mathrm{m}}$ is a mix modulus if there is more than one constituent which can be modified when dealing with various type of clay as illite has higher density number rather than owned by kaolinite and smectite. Therefore, clay's content in sand can give lower or higher bulk modulus.

- Cement is also a part of media which can lock the grain contact, increasing the stiffness and fastening the wave propagation through the media.

Saturated rock modulus is a function of water saturation which is written in Eq. (3) while water saturation is also affected by porosity as defined by Archie (1941). Dry rock modulus which represents rock bulk modulus is related to tomineral modulus, porosity, and pore space stiffness (Avseth, Mukerji, \& Mavko, 2005). Avsethet. al. (2005) definesspore space stiffness as:

$$
\frac{1}{K_{\varnothing}}=\frac{1}{V_{\text {pore }}} \frac{\partial V_{\text {pore }}}{\partial \sigma}
$$

where $\mathrm{V}_{\text {pore }}$ is pore volume, and $\sigma$ is the differential between hydrostatic confining stress from the passing wave.

In this study, clay volume is calculated from Gamma Ray log, porosity log is estimated from density log, while water saturation is derived from resistivity log. Then, water saturation calculation is corrected by shale distribution analysis. Picket plot is used to obtain tortuosity factor (a), cementation exponent (m), and saturation exponent (n). Data has indicated that quartz and clay are typical mineralogy found and analyzed through XRD in the observed sand, where the types of clay mineral which are found based on XRD are kaolinite and illite.

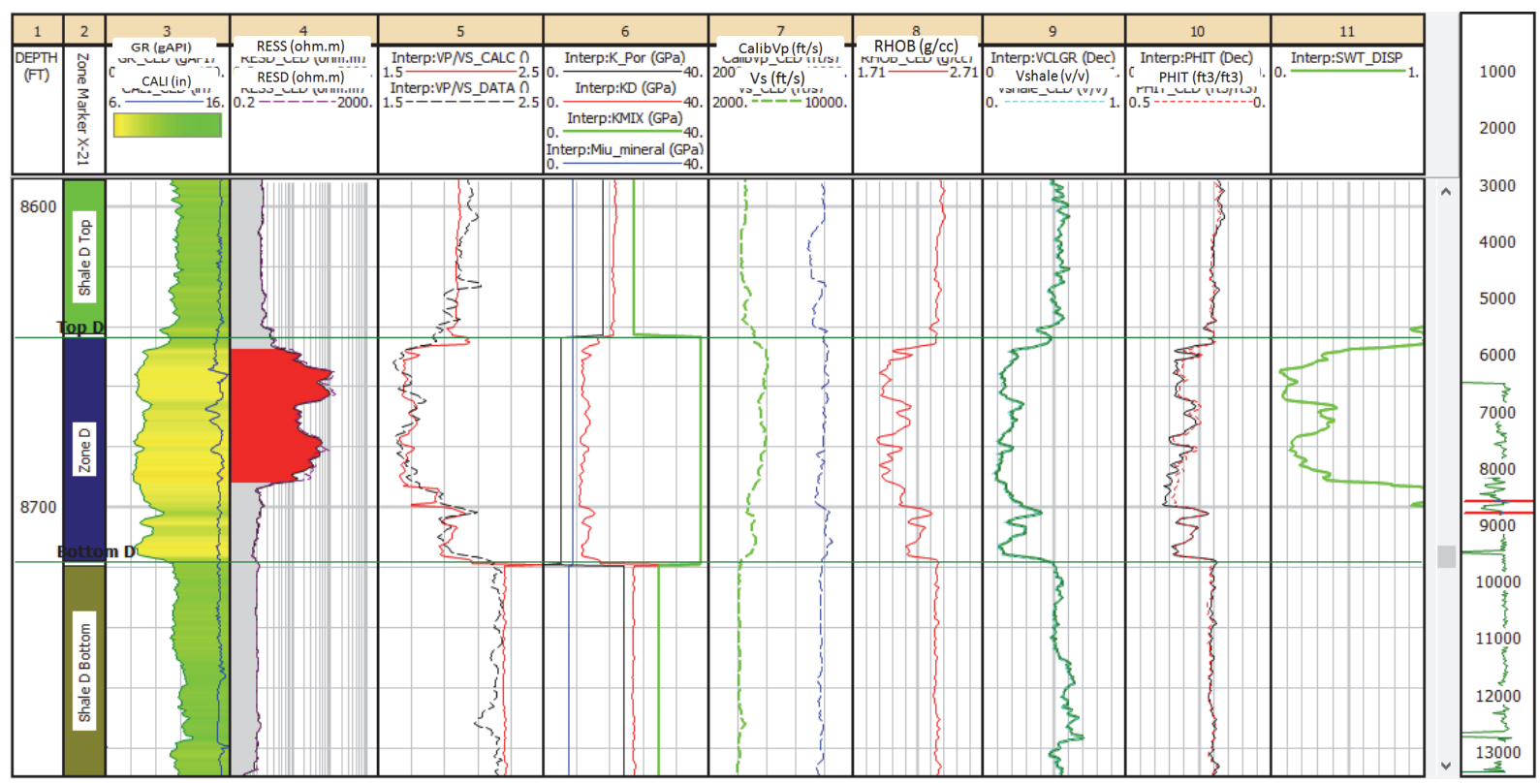

Figure 6. Calculated $\mathrm{Vp} / \mathrm{Vs}$ (red solid line) by analytical equation which is plotted in track 5 in Well X-21 at Zone D 
Then, P-wave and S-wave velocity ratios which are using Eq. (10). Yet, there are some variables which are assumed to be constant value. Hydrostatic pressure relative constant which causes $K_{\text {pore }}$ constant. $K_{d}, K_{m}, K_{f}, a-m-n, R_{w}$ are considered constants for having common condition within a dispersed shaly sand-body interval. The only variation hence is fluid saturation against depth which describes in $\mathrm{R}_{\mathrm{t}}$ values and Porosity. This is an example of $\mathrm{Vp} / \mathrm{Vs}$ calculation result at well X-21 in which the zone has gas-water contact contrast. There is no core image to validate the model, which is why confidence level is still lower compared to the well which owns whole core images (Fig.6). The results of other calculations are included in appendix.

From Thomas-Stieber's curve, it shows that shale texture is laminated or dispersed. The curve shows that dispersed shale makes the porosity shrink drastically as the clay volume increases in the pores. In contrast, laminated shale affect decrease in porosity by increasing clay volume but it is not too drastic. This case can affect seismic wave diffusion. When porosity is smaller due to clay volume, seismic velocity will be higher. Figure 7 is the example of Thomas-Stieber's curve based on the data.

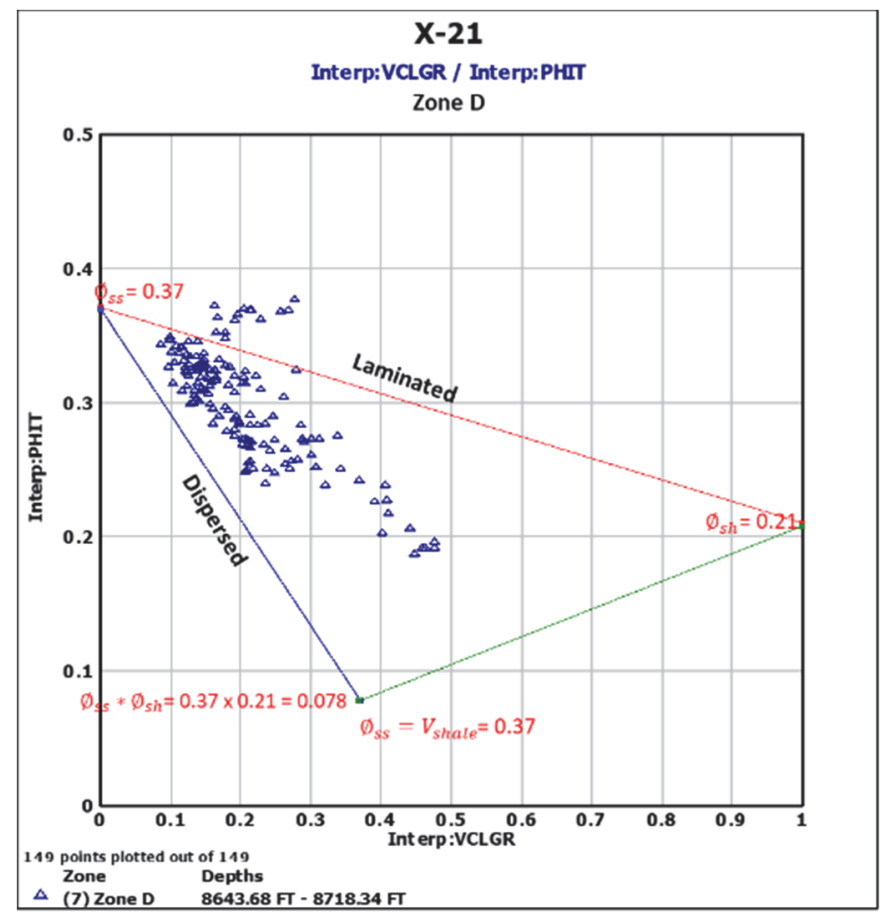

(a)

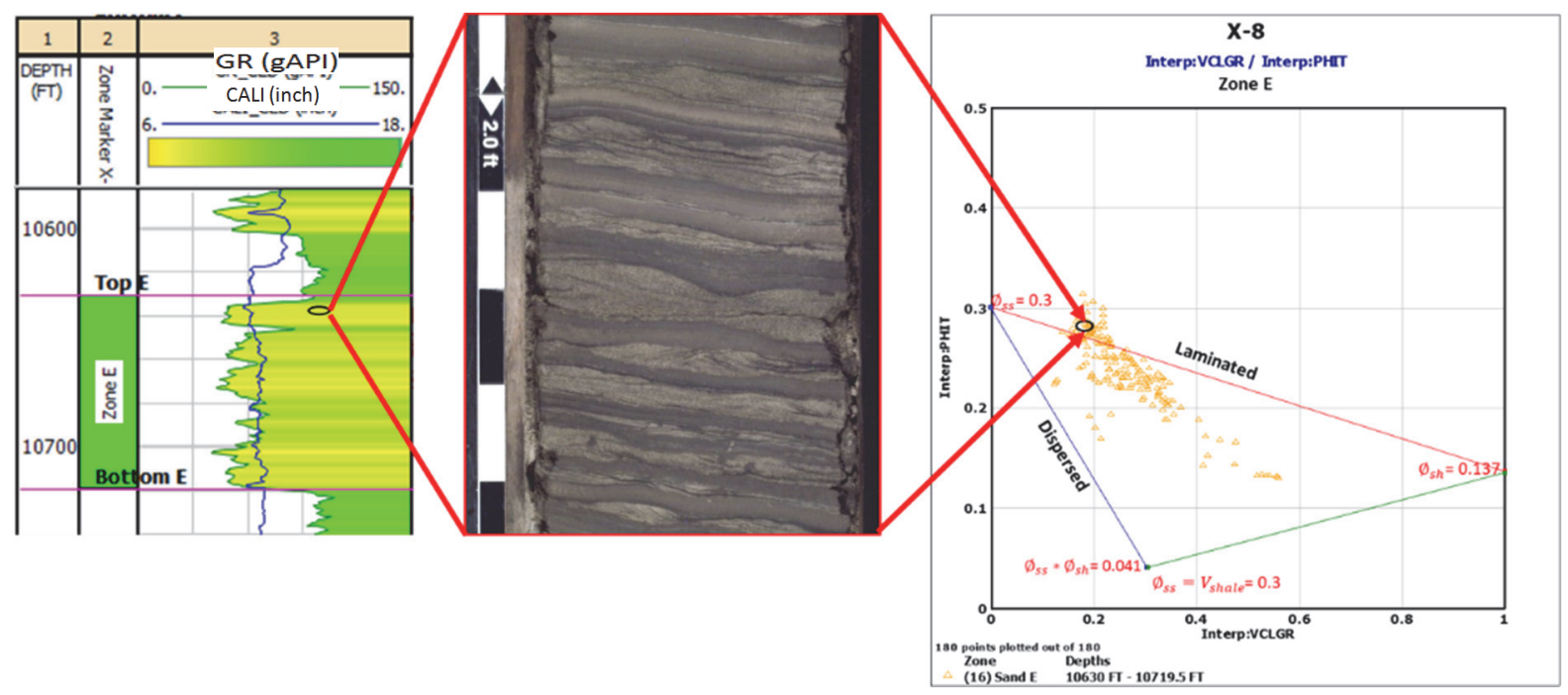

(b)

Figure 7. Thomas-Stieber plot in shaly sand reservoir, (a) at Sand D of well X-21and (b) at Sand E of well X-8 


\section{Discussion}

Shale texture interpretation based on Thomas-Stieber crossplot is validated with core image data. Figure $7 \mathrm{~b}$ indicates that shale texture in sand zone of X-8 is laminated shale. Core image also displays laminated shale, so the interpretation is matched with core data.

Water saturation also should be corrected by shale texture as it can be higher when shale texture is laminated compared to dispersed, especially in modeling seismic velocity against water saturation. As exposed in Fig.7, for example in the same porosity e.g. $25 \%$, shale volume will be greater in laminated shale i.e. $37 \%$ compared to the dispersed shale which is only $9 \%$, so that water saturation will be greater in larger shale volume in the pores. The large water saturation, the softer rock, and the resistivity shrink so that $\mathrm{Vp}$ becomes large. $\mathrm{Vp} / \mathrm{Vs}$ to $\mathrm{Sw}$ model which are related to the type of shale texture is provided in Fig.7.

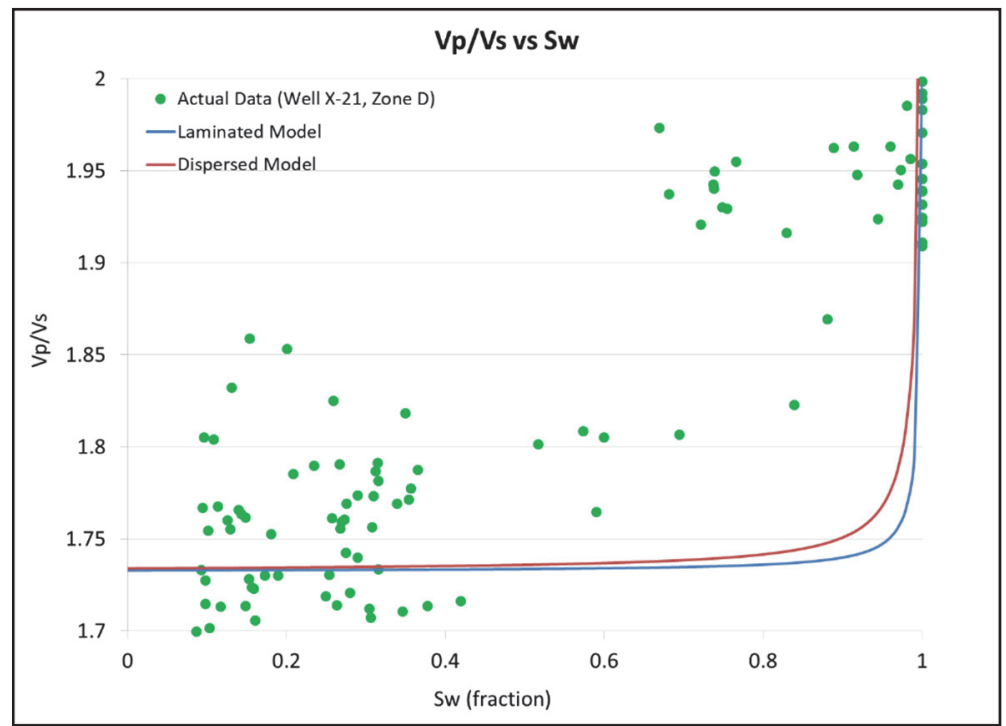

Figure $8 . \mathrm{Vp} / \mathrm{Vs}$ model and actual data from Zone D of Well X-21

From Fig.8, The actual data are fall in dispersed model. It is appropriate with Thomas-Stieber's curve in Fig.7 (a) which also shows that the data of zone $\mathrm{D}$ in well $\mathrm{X}-21$ is dispersed shale. The actual data represents that laminated and dispersed shale give different $\mathrm{Vp} / \mathrm{Vs}$ values.

Decrease in porosity is quite drastic for dispersed shale and will affect the response of seismic wave velocity. Based on Eq. (8), if porosity is high, P-wave and S-wave velocity ratios will also becomehigh, so that it makes the $\mathrm{P}$-wave velocity high. It means that when pore medium is quite tight, $\mathrm{P}$-wave can spread quickly through the pore medium.

In predicting $V p / V s$ based on analytical equation, the value of $\mathrm{K}_{\mathrm{m}}, \mathrm{K}_{\text {pore }}, \mathrm{K}_{\mathrm{f}}$ and $\mu_{\mathrm{m}}$ are assumed due to the limitation data. In this research, actually, there is nodirect measure from core analysis at laboratory since there is no such equipment in country which has laboratory unit to measure elastic parameters in such reservoir pressure. The values are assumed using sensitivity analyzer tool (Table 1) and matched with the literature (Schon, 2011).

Table 1 . The sensitivity parameters result by analyzer tool

\begin{tabular}{|c|c|c|c|c|c|c|c|c|c|c|c|c|c|c|c|c|}
\hline \multirow{2}{*}{ Well } & \multirow{2}{*}{ Zone } & \multirow{2}{*}{ Indication } & \multirow{2}{*}{ Kh (Gpa) } & \multicolumn{9}{|c|}{ Sand } & \multicolumn{2}{|c|}{ Clay above } & \multicolumn{2}{|c|}{ Clay below } \\
\hline & & & & $\mu q(\mathrm{Gpa})$ & Km (Gpa) & Kd (Gpa) & Kw (Gpa) & Kpor (Gpa) & $\mathbf{A R}$ & Sensitivity & Avg. Error & Texture & Kc (Gpa) & $\mu \mathrm{c}$ (Gpa) & Kc (Gpa) & $\mu \mathrm{c}$ (Gpa) \\
\hline$X-21$ & D & Gas-Water & 0.192 & 7 & 38 & 10 & 4 & 4.07 & 0.11 & $14 \%$ & $2 \%$ & Dispersed & 22 & 7 & 28 & 6 \\
\hline$x-1$ & B & Gas-Water & 0.35 & 11 & 28 & 15 & 4 & 8.08 & 0.29 & $5 \%$ & $1 \%$ & Laminated & 23 & 7 & 23 & 7 \\
\hline$x-7$ & $B$ & Gas-Water & 0.3 & 7 & 35 & 9 & 4 & 3.72 & 0.11 & $19 \%$ & $4 \%$ & Laminated & 25 & 5 & 21 & 7 \\
\hline$x-8$ & $\mathrm{D}$ & Gas-Water & 0.3 & 9 & 38 & 15 & 4 & 8.00 & 0.21 & $8 \%$ & $9 \%$ & Laminated & 22 & 6 & 25 & 6 \\
\hline $\mathrm{X}-8$ & $\mathrm{E}$ & Gas-Water & 0.3 & 20 & 25 & 18 & 4 & 18.64 & 0.75 & $2 \%$ & $14 \%$ & Laminated & 20 & 9 & 27 & 6 \\
\hline$x-8$ & $\mathrm{C}$ & Water & 0.3 & 7 & 38 & 19 & 4 & 12.24 & 0.32 & $4 \%$ & $3 \%$ & Laminated & 25 & 6 & 24 & 6 \\
\hline$x-1$ & $C \& D$ & Gas & 0.2 & 7 & 37 & 15 & 4 & 4.74 & 0.13 & $13 \%$ & $9 \%$ & Laminated & 21 & 7 & 21 & 10 \\
\hline $\mathrm{X}-8$ & $\mathrm{BC}$ & Gas & 0.35 & 6 & 38 & 18 & 4 & 11.18 & 0.29 & $7 \%$ & $3 \%$ & Laminated & 25 & 6 & 24 & 6 \\
\hline $\mathrm{X}-21$ & $C$ & Gas & 0.3 & 7 & 37 & 10 & 4 & 4.11 & 0.11 & $13 \%$ & $4 \%$ & Laminated & 22 & 7 & 22 & 7 \\
\hline$x-21$ & B & Gas & 0.3 & 6 & 28 & 9 & 4 & 3.98 & 0.14 & $13 \%$ & $3 \%$ & \begin{tabular}{|l|} 
Laminated \\
\end{tabular} & 25 & 6 & 24 & 7 \\
\hline$X-21$ & A & Gas & 0.3 & 13 & 35 & 10 & 4 & 4.11 & 0.11 & $10 \%$ & $1 \%$ & Dispersed & 21 & 8 & 23 & 7 \\
\hline
\end{tabular}


In example, Fig. 8 shows the parameter sensitivity resultusing the analyzer tool for Zone B of Well X-1. The sensitivity analyzer tool produces $K_{m}$ of $28 \mathrm{GPa}$ to get the $\mathrm{Vp} / \mathrm{Vs}$ match with(Fig.9). It is matched with The GR $\log$ which describes that Zone B is shaly sand enough. The $\mathrm{K}_{\mathrm{m}}$ for quartz is $38 \mathrm{GPa}$ while $\mathrm{K}_{\mathrm{m}}$ for clay is about 1.5 - $39 \mathrm{GPa}$. Nevertheless, in this case, $\mathrm{K}_{\mathrm{m}}$ for shale zone in the top and the bottom of Sand B is $23 \mathrm{GPa}$. Therefore, $\mathrm{K}_{\mathrm{m}}$ for Sand B is still reasonable which is $28 \mathrm{GPa}$. From Thomas-Stieber's curve for this zone (Fig.10), it shows that the shale texture is laminated, so that the used $\mathrm{Sw}$ model for predicting $\mathrm{Vp} / \mathrm{Vs}$ is laminated.

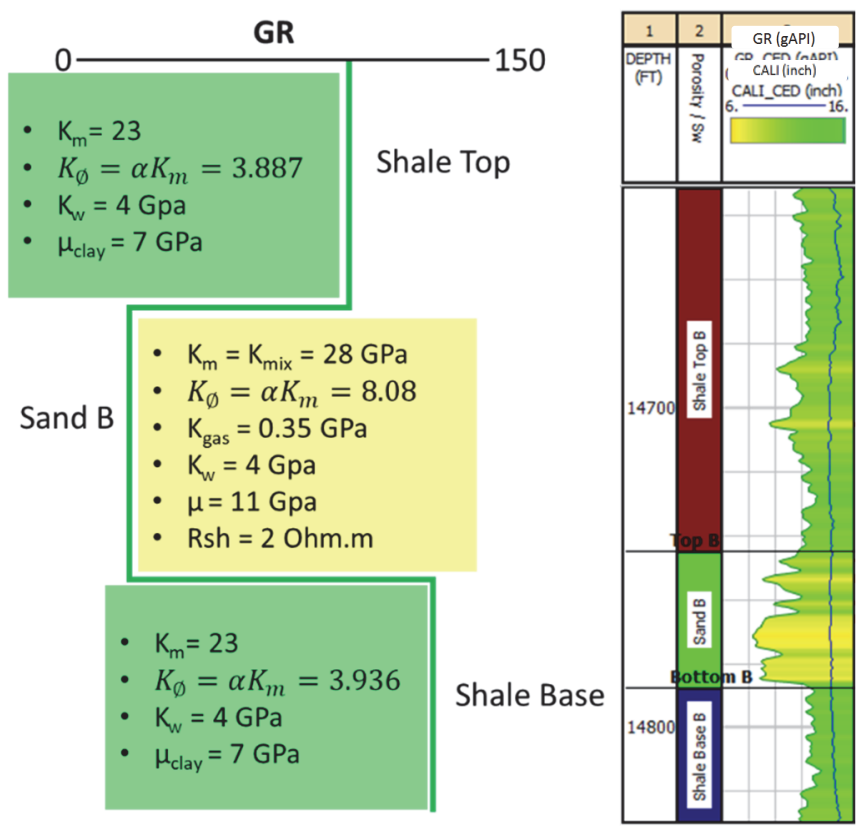

Figure 9. The sensitivity parameters result for Zone B in Well X-1

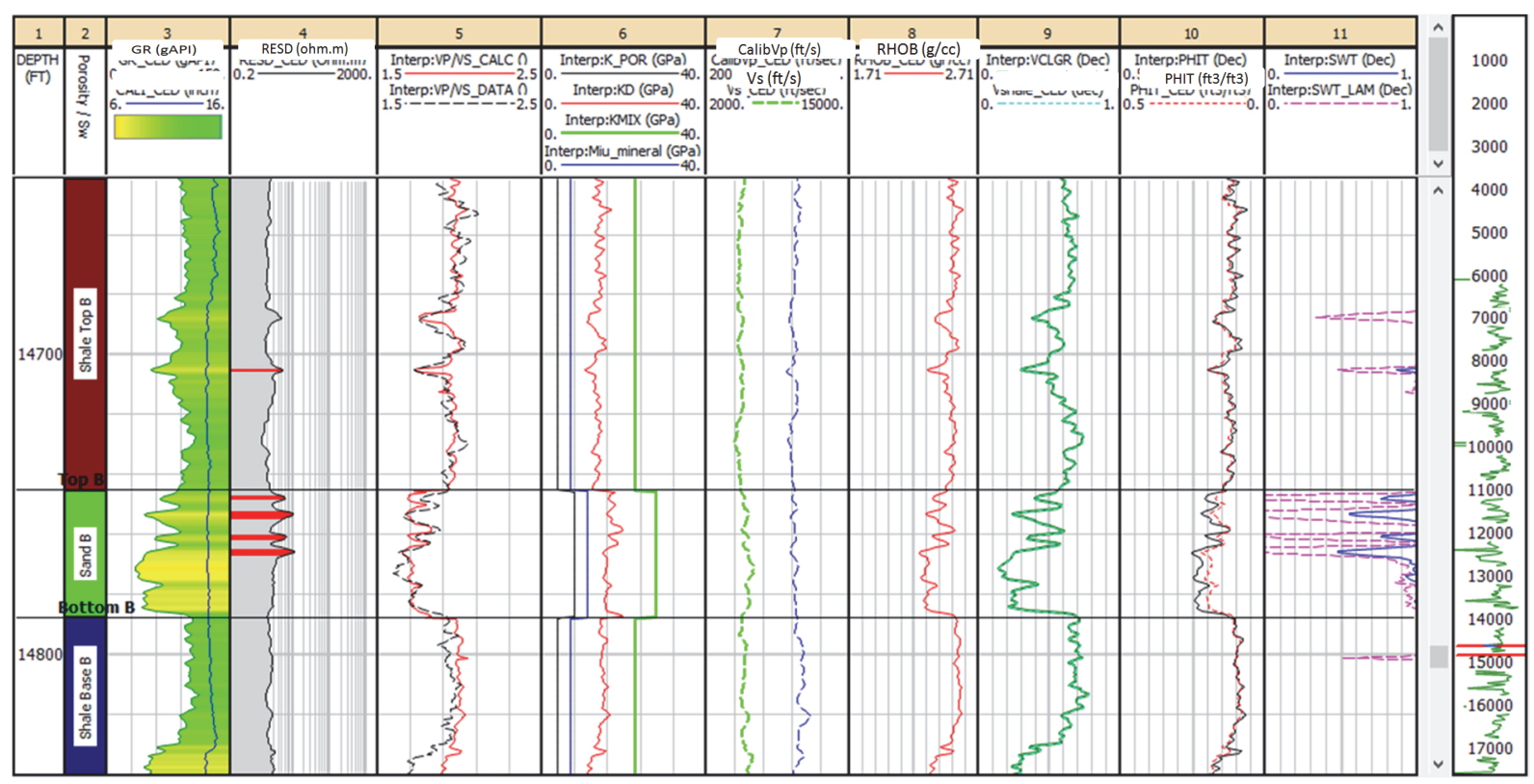

Figure 10. Plotting $\mathrm{Vp} / \mathrm{Vs}$ prediction (red line) with $\mathrm{Vp} / \mathrm{Vs}$ data (black line) is in track 5 for Zone $\mathrm{B}$ of Well $\mathrm{X}-1$ 


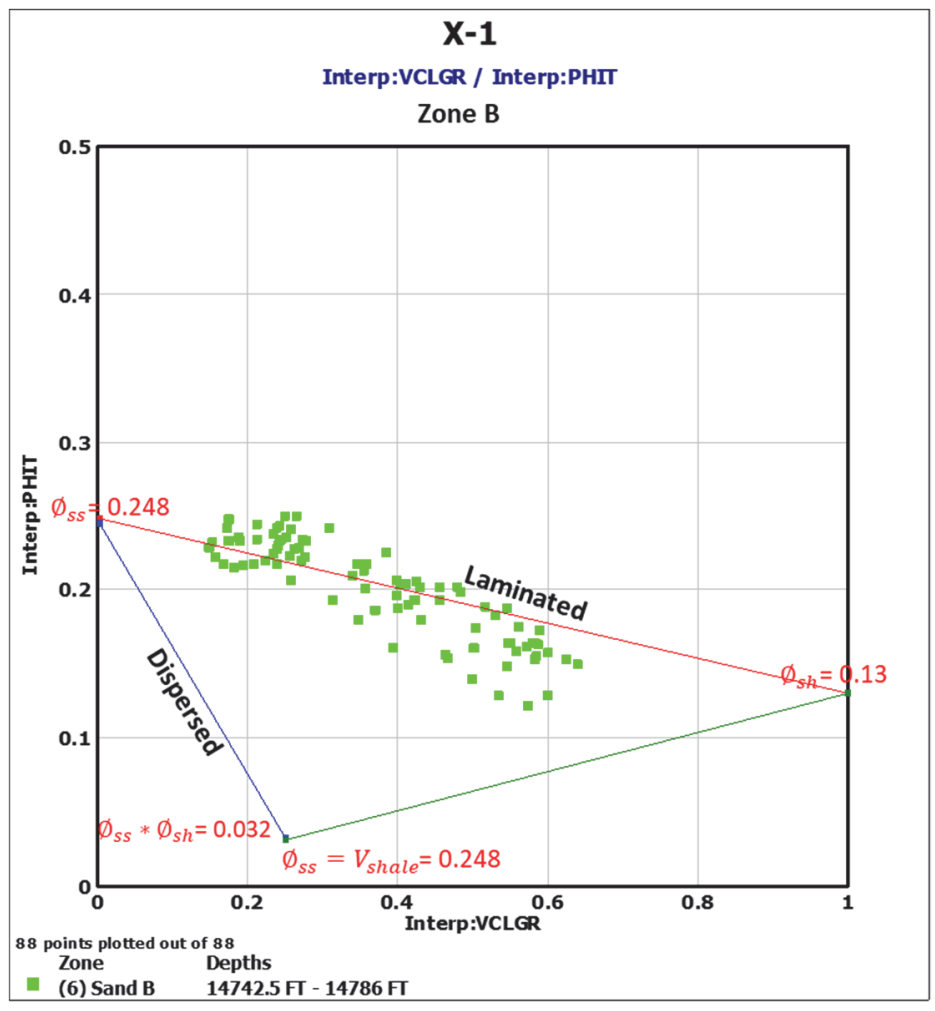

Figure 11. Thomas-Stieber's'scurve for Zone B in Well X-1

Actually, $\mathrm{K}_{\mathrm{m}}$ is influenced by constituent (fraction of each mineral). It can be represented by shale volume while sand volume is $(1-V s h)$. When shale volume is high, $K_{m}$ becomes low. $K_{m}$ can be calculatedusing Voight and Reuss method as written in Eq. (15). It is accepted to input a value of $\mathrm{K}_{\mathrm{m}}$ for one layer of reservoir when a reservoir has almost homogeneous lithology. On the other hand, when $\mathrm{Km}$ is assumed to be constant, it can be covered by high $\mathrm{K}_{\mathrm{d}}$ and low $\mathrm{K}_{\text {pore }}$ as related to the following equation.

$$
\frac{1}{K_{d}}=\frac{1}{K_{m}}+\frac{\emptyset}{K_{\varnothing}}
$$

Based on Eq. (16), $\mathrm{K}_{\text {pore }}$ is related topore volume and pore pressure. When pore pressure is reducedd by production activity, $\mathrm{K}_{\text {pore }}$ will be high. In contrast, when pore pressure is high due to aquifer, migration or injection phenomena, $\mathrm{K}_{\text {pore }}$ will be low. Meanwhile, $\mathrm{K}_{\mathrm{d}}$ can be changed due to porosity. Therefore, $\mathrm{K}_{\mathrm{m}}$ might be constant. The Gassmann's equation is valid for thethe connected pores as $\mathrm{Vp}$ is the assumption of those parameters which can still be accepted.

\section{Conclusion}

a) This study has proven the hypothesis of Vp/Vs relationship with True Resistivity and dependent of shale distribution in pore space - shaped dominantly by quartz grain in deepwater turbidite deposits.

b) A quantitative interpretation now can be realized to predict $\mathrm{Vp} / \mathrm{Vs}$ from True Resistivity or Gas Saturation and inversely a promising formula also can be established in predicting Gas Saturation and True Resistivity from $\mathrm{Vp} / \mathrm{Vs}$.

c) The established formula is derived by joining Gassmann's and Archie's equations at the same time, taking all assumptions as if as real rock samples are set in ideal case (homogen, isotropic, consolidated sand, highly fluid saturated). Mixing Law is one ofsupporting method which allows in-situ rock complexity meet assumption criteria such as homogenize rock which is considered above.

d) Shale texture also takes important role during forward modeling and $\mathrm{Vp} / \mathrm{Vs}$ calculation take place, since it influences the value of porosity, water saturation, and $\mathrm{K}_{\mathrm{m}}$. From the exercised model and real data, those have shown that laminated shaly sand gives lower $\mathrm{Vp} / \mathrm{Vs}$ response thandispersed shaly sand. Therefore, it leads to an understanding of having dispersed shaly sand to be more sensitive to discriminate gas from water. 


\section{Reference}

Archie, G. (1941). The Electrical Resistivity Log as an Aid in Determining Some Reservoir Characteristics. Dallas Meeting. https://doi.org/10.2118/942054-G

Avseth, P., Mukerji, T., \& Mavko, G. (2005). Quantitative Seismic Interpretation. Cambridge: Cambridge University Press. https://doi.org/10.1017/CBO9780511600074

Gassmann, F. (1951). Elastic Waves Through a Packing of Spheres. Geophysics, 16, 673-685. https://doi.org/10.1190/1.1437859

Mavko, G., Mukerji, T., \& Dvorkin, J. (2009). The Rock Physics Handbook, Second Edition. Cambridge: Cambridge University Press. https://doi.org/10/1017/CBO9780511626753

Mendrofa, D. M. F. (2006). An Analytical Derivation of Resistivity from Acoustic Impedance in Porous Elastic Media: M. S. Thesis, University of Indonesia.

Schon, J. (2011). Physical Properties of Rocks. Oxford: Elsevier.

Thomas, E., \& Stieber, S. (1975). The Distribution of Shale in Sandstones and its Effect Upon Porosity. SPWLA Sixteenth Annual Logging Symposium. SPWLA.

Tyagi, A. K., Guha, R., Voleti, D., \& Saxena, K. (2009). Challenges in The Reservoir Characterization of a Laminated Sand Shale Sequence. 2nd SPWLA-India Symposium. India: SPWLA.

\section{Copyrights}

Copyright for this article is retained by the author(s), with first publication rights granted to the journal.

This is an open-access article distributed under the terms and conditions of the Creative Commons Attribution license (http://creativecommons.org/licenses/by/4.0/). 